Proyecciones Journal of Mathematics

Vol. 38, No 2, pp. 315-324, June 2019.

Universidad Católica del Norte

Antofagasta - Chile

\title{
On the uniform ergodic theorem in invariant subspaces
}

\author{
Abdelaziz Tajmouati \\ Sidi Mohamed Ben Abdellah University, Morocco \\ Abdeslam El Bakkali \\ Chouaib Doukkali Univesity, Morocco \\ and \\ Fatih Barki \\ Sidi Mohamed Ben \\ Received : October 2017. Accepted : March 2019
}

\begin{abstract}
Let $T$ be a bounded linear operator on a Banach space $X$ into itself.

In this paper, we study the uniform ergodicity of the operator $\left.T\right|_{Y}$ when $Y$ is a closed subspace invariant under $T$. We show that if $T$ satisfies $\lim _{n \rightarrow \infty} \frac{\left\|T^{n}\right\|}{n}=0$, then $T$ is uniformly ergodic on $X$ if and only if the restriction of $T$ to some closed subspace $Y \subset X$, invariant under $T$ and $R\left[(I-T)^{k}\right] \subset Y$ for some integer $k \geq 1$, is uniformly ergodic. Consequently, we obtain other equivalent conditions concerning the theorem of Mbekhta and Zemànek [9, theorem 1], also to the theorem of the Gelfand-Hille type.
\end{abstract}

2010 Mathematics Subject Classification. 47A35, 47A25, 47A15.

Key words and phrases. Uniform ergodic theorem, Cesàro averages, decomposition ergodic. 


\section{Introduction}

Throughout this paper, $\mathbf{B}(X)$ denotes the Banach algebra of all bounded linear operators on a Banach space $X$ into itself. For $T \in \mathbf{B}(X)$, we denote by $R(T), N(T), \sigma(T), r(T)$ and $\rho(T)$, the range, the kernel, the spectrum, the spectral radius and the resolvent set of $T$, respectively. A closed subspace $Y$ of $X$ is called invariant under $T$ or shortly $T$-invariant if $T(Y) \subset Y$.

By $M_{n}(T)=\frac{1}{n} \sum_{k=0}^{n-1} T^{k}$, for $n \in N$, denote the sequence of the arithmetic means of the powers of $T$. An operator $T \in \mathbf{B}(X)$ is called uniformly ergodic (resp. mean ergodic) if $\left\{M_{n}(T)\right\}$ is uniformly (resp. strongly) convergent in $\mathbf{B}(X)$.

Recall that, $T \in \mathbf{B}(X)$ is called Cesàro bounded if $\sup _{n}\left\|M_{n}(T)\right\|<\infty$. Then a every uniformly ergodic operator $T$ is necessarily Cesàro bounded, and $\frac{\left\|T^{n}\right\|}{n} \rightarrow 0$ when $n \rightarrow \infty$, by the following identity:

$$
T^{n}=(n+1) M_{n+1}(T)-M_{n}(T) .
$$

In 1938, Yosida [12] showed that when $\left\{M_{n}(T)\right\}$ converge strongly to $P \in \mathbf{B}(X)$ (i.e. $T$ is mean ergodic) then $P$ is the projection onto the space $N(I-T)$ along $\overline{R(I-T)}$, corresponding to the ergodic decomposition (see [7, Theorem 2.1.3.])

$$
X=N(I-T) \oplus \overline{R(I-T)} .
$$

Much of ergodic operator theory is concerned with determining when, conversely, the convergence to 0 of $\left\{\frac{T^{n}}{n}\right\}$ and/or the boundedness of $\left\{M_{n}(T)\right\}$ implies the convergence of $\left\{M_{n}(T)\right\}$ in the operator topology considered. A fundamental theorem due to M. Lin [8] (see also [7, Theorem 2.2.1. p.87]) says that when $\frac{\left\|T^{n}\right\|}{n} \rightarrow 0, T$ is uniformly ergodic if and only if $R(I-T)$ is closed. In this case $R\left[(I-T)^{k}\right]$ is closed for every integer $k \geq 1$.

Mbekhta and Zemànek [9], still assuming $\frac{\left\|T^{n}\right\|}{n} \rightarrow 0$ when $n \rightarrow \infty$, have showed that $T$ is uniformly ergodic if and only if $R\left[(I-T)^{k}\right]$ is closed for some integer $k \geq 1$. The case $k=2$, is due to Dunford [4]. On the other hand, Koliha in [6, Theorem 4] proved that $\left\{T^{n}, n \geq 0\right\}$ converge uniformly if and only if $r(T) \leq 1, \sigma(T) \cap \Gamma \subset\{1\}$ and 1 is a pole of the resolvent of order at most 1 ( $\Gamma$ is the unit circle). The latter condition is equivalent to uniform ergodicity (see [4]). In the same case, Mbekhta and Zemànek give another condition equivalent to Koliha's theorem [6], presented in the following theorem: 
Theorem 1.1. [9, Corollary 3] Let $T$ be a bounded linear operator on a complex Banach space $X$. Then the following conditions are equivalent:

1. $\left\{T^{n}\right\}_{n \geq 0}$ converge uniformly in $\mathbf{B}(X)$,

2. $T$ is uniformly ergodic and $\sigma(T) \cap \Gamma \subset\{1\}$,

3. $\lim _{n \rightarrow \infty} \frac{\left\|T^{n}\right\|}{n}=0, \sigma(T) \cap \Gamma \subset\{1\}$ and $R\left[(I-T)^{k}\right]$ is closed for some integer $k \geq 1$,

4. $\left\|T^{n}-T^{n+1}\right\| \rightarrow 0$ when $n \rightarrow \infty$ and $R\left[(I-T)^{k}\right]$ is closed for some integer $k \geq 1$.

M. E. Becker [2] gives an example of a subspace of $X$ which is invariant under $T$ denoted by $X_{1}:=\left\{x \in X: \lim _{n \rightarrow \infty} \sum_{k=1}^{n} \frac{T^{k} x}{k}\right.$ exists $\}$. By the Hahn-Banach theorem, $X_{1} \subset \overline{R(I-T)}$. She proved [2, Remark 2] that if $\lim _{n \rightarrow \infty} \frac{\left\|T^{n}\right\|}{n}=0$ and $R(I-T) \subset X_{1}$, then $T$ is uniformly ergodic if and only if $X_{1}$ is closed. When $T$ is power-bounded, $R(I-T) \subset X_{1}$.

This paper is organized as follows. In section 2, we give some definitions and fundamental properties of ergodic operator theory. In section 3, we show that for every operator $T \in \mathbf{B}(X)$ which satisfies $\lim _{n \rightarrow \infty} \frac{\left\|T^{n}\right\|}{n}=0$, $T$ is uniformly ergodic if and only if there exists a closed subspace $Y \subset$ $X, T$-invariant, contains $R\left[(I-T)^{k}\right]$ for some integer $k \geq 1$ and $\left.T\right|_{Y}$ is uniformly ergodic. This result was also obtained for an operator satisfying $\lim _{n \rightarrow \infty} \frac{T^{n} x}{n}=0$ for all $x \in X$, in the weak operator topology.

\section{Preliminaries}

In this section, we briefly review the definitions and some basic properties which we will need in the sequel.

For $T \in \mathbf{B}(X)$, Recall that the ascent of $T$ is the smallest non negative integer $n$ such that $N\left(T^{n}\right)=N\left(T^{n+1}\right)$, if no such $n$ exists, we write $\operatorname{asc}(T)=\infty$. Similarly, the descent of $T$ is the smallest nonnegative integer $n$ such that $R\left(T^{n}\right)=R\left(T^{n+1}\right)$, if there is no such n, we write $\operatorname{des}(T)=\infty$ (see e.g. [1, Definition 3.1] or [3] p.10 ). It may be instructive to note that one may have $\operatorname{des}(T) \leq n<\infty$ without $R\left(T^{n}\right)$ begin closed (see example at the end of [5]). We mention the following characterization: 
Lemma 2.1. [6, Lemma 1.1] Given a non-negative integer $d$ and $T \in$ $\mathbf{B}(X)$, we have

(i) $\operatorname{asc}(T) \leq d<\infty$ if and only if $R\left(T^{d}\right) \cap N\left(T^{m}\right)=\{0\}$, for some (equivalently, all) integer $m \geq 1$.

(ii) des $(T) \leq d<\infty$ if and only if $R\left(T^{m}\right)+N\left(T^{d}\right)=X$, for some (equivalently, all) integer $m \geq 1$.

If both $\operatorname{asc}(T)$ and $\operatorname{des}(T)$ are finite, then they are equal, and $X=$ $R\left(T^{d}\right) \oplus N\left(T^{d}\right)$

where $d=\operatorname{asc}(T)=\operatorname{des}(T)$. For more details see Lemma 1.4.6, Lemma 3.2.4 and Proposition 1.4.3 from [3] or p.330 from [11].

Lemma 2.2. Let $T$ be a bounded linear operator on a real or complex Banach space $X$. Let $Y \subset X$ be a closed subspace which is $T$-invariant and $\left.T\right|_{Y}$ be the restriction of $T$ to $Y$.

If $T$ is uniformly ergodic (resp. mean ergodic), then $\left.T\right|_{Y}$ is uniformly ergodic (resp. mean ergodic).

Proof:

Let $Y \subset X$ be a closed subspace which is T-invariant, and denote $S=\left.T\right|_{Y}$. Since $Y$ is T-invariant, it is also $T^{n}$-invariant for each $n \in N$, thus it is also invariant under $S_{n}=\left(\left.T^{n}\right|_{Y}\right)$. Using that $Y$ is closed, we get that both kind of limits are inside of $Y$.

Thus, $S$ is uniformly ergodic.

Proposition 2.1. [5, Remark 1.4] Let $T$ be a bounded linear operator on a complex Banach space $X$. If $T$ satisfies either of the following assumptions:

(i) $T$ is Cesàro bounded operator, or

(ii) $\frac{T^{n}}{n}$ converge weakly to 0 .

Then the spectral radius of $T$ is not greater than 1 . Moreover, $N(I-$ $T) \cap R(I-T)=\{0\}$, which in turn yields $\operatorname{asc}(I-T) \leq 1$.

The next lemma can be considered a version of the Gelfand-Hille theorem [13].

Lemma 2.3. [9, Corollary 2] Let $T$ be a bounded linear operator on a complex Banach space $X$ such that $\sigma(T)=\{1\}$. If $T$ is uniformly ergodic, then $T=I$. 


\section{Main results}

The first main result of this paper is the following theorem:

Theorem 3.1. Let $T$ be a bounded linear operator on a real or complex Banach space $X$ such that $\lim _{n \rightarrow \infty} \frac{\left\|T^{n}\right\|}{n}=0$. Let $\left.T\right|_{Y}$ be the restriction of $T$ to a closed subspace $Y \subset X$, which is invariant under $T$, and $R(I-T) \subset Y$.

If $\left.T\right|_{Y}$ is uniformly ergodic, then $T$ is uniformly ergodic.

\section{Proof:}

Let $Y$ be a closed subspace of $X$ (not trivial) which is invariant under $T$, assume that $R(I-T)$ is included in $Y$, and suppose that $\left.T\right|_{Y}$ is uniformly ergodic.

Put $Z:=\overline{R(I-T)}$. Then $Z \subset Y$, so $\left.T\right|_{Z}$ is uniformly ergodic on $Z$. The limit is 0 on $R(I-T)$, so by continuity of the limit $\left\|M_{n}\left(\left.T\right|_{Z}\right)\right\| \rightarrow 0$. Hence $\left(I_{Z}-\left.T\right|_{Z}\right) Z=Z$. Then

$$
R(I-T) \subset Z=\left(I_{Z}-\left.T\right|_{Z}\right) Z \subset R(I-T),
$$

which implies $R(I-T)=Z$, so $R(I-T)$ is closed and $T$ is uniformly ergodic by [8]. $\square$

Corollary 3.1. Let $T$ be a bounded linear operator on a complex Banach space $X$ such that $\lim _{n \rightarrow \infty} \frac{\left\|T^{n}\right\|}{n}=0$. Let $Y \subset X$ be a closed subspace $T$ invariant such that $R\left[(I-T)^{k}\right] \subset Y$, for some integer $k \geq 1$. If $\left.T\right|_{Y}$ is uniformly ergodic, then $T$ is uniformly ergodic.

\section{Proof:}

Let $Y \subset X$ be a closed subspace T-invariant such that $R\left[(I-T)^{k}\right] \subset$ $Y$, for some integer $k \geq 1$. Let's denote $S=\left.T\right|_{Y}$, and assume that $S$ is uniformly ergodic. By uniform ergodicity, $Y=R(I-S) \oplus N(I-S)$ with $R(I-S)$ closed [8].

We will show $R\left[(I-T)^{k}\right]=R(I-S)$. Since $R\left[(I-S)^{k}\right] \subset R\left[(I-T)^{k}\right]$, we need to prove only $R\left[(I-T)^{k}\right] \subset R\left[(I-S)^{k}\right]$. Since $R\left[(I-T)^{k}\right] \subset Y=$ $R(I-S) \oplus N(I-S)$ by assumption, for $x \in X$ write $(I-T)^{k} x=(I-S) y+z$ with $y, z \in Y$ and $T z=S z=z$. Then

$$
M_{n}(T)(I-T)^{k} x=M_{n}(S)(I-S) y+z,
$$


and $\left\|T^{n}\right\| / n \rightarrow 0$ yields that $z=0$, so $R\left[(I-T)^{k}\right] \subset R(I-S)$. But by uniform ergodicity $I-S$ is invertible on $R(I-S)$, which is closed, so $R\left[(I-S)^{k}\right]=R(I-S)$.

Hence $R\left[(I-T)^{k}\right] \subset R\left[(I-S)^{k}\right]$ and equality holds, so $R\left[(I-T)^{k}\right]=$ $R(I-S)$ is closed.

Hence $T$ is uniformly ergodic by [9].

In the following theorem, we give a generalization of Theorem 3.1 and Corollary 3.1 where $X$ is a real or complex Banach space and $T \in \mathbf{B}(X)$ satisfying $\lim _{n \rightarrow \infty} \frac{T^{n} x}{n}=0$, for all $x \in X$, in the weak operator topology.

Theorem 3.2. Let $T$ be a bounded linear operator on a real or complex Banach space $X$ such that $\lim _{n \rightarrow \infty} \frac{T^{n} x}{n}=0$, for all $x \in X$, in the weak operator topology. Let $Y \subset \stackrel{n \rightarrow \infty}{X}$ be a closed subspace $T$-invariant such that $R\left[(I-T)^{k}\right] \subset Y$, for some integer $k \geq 1$.

If $\left.T\right|_{Y}$ is uniformly ergodic, then $T$ is uniformly ergodic.

\section{Proof:}

Let $Y \subset X$ be a closed subspace T-invariant such that $R\left[(I-T)^{k}\right] \subset$ $Y$, for some integer $k \geq 1$. Let's denote $S=\left.T\right|_{Y}$, and assume that $S$ is uniformly ergodic.

Using the technique employed in the proof of Corollary 3.1, we get $R\left[(I-T)^{k}\right]=R(I-S)$. Now, we prove that if $k>1$, then also $R[(I-$ $\left.T)^{k-1}\right]=R(I-S)$.

Let $x \in X$, then $(I-T)^{k} x=(I-S) y=(I-T) y$ for some $y \in$ $Y$, and by uniform ergodicity of $S$, we can take $y \in R(I-S)$. Then $(I-T)\left[(I-T)^{k-1} x-y\right]=0$, so $(I-T)^{k-1} x=y+z$ with $y \in R(I-S)$ and $z \in N(I-T)$.

Applying $M_{n}(T)$ to both sides and using $T^{n} / n \rightarrow 0$ weakly, we get $z=0$, which implies $R\left[(I-T)^{k-1}\right] \subset R(I-S)$. We proceed by induction $R(I-T)=R(I-S)$.

Then $M_{n}\left(\left.T\right|_{R(I-T)}\right)=M_{n}\left(\left.S\right|_{R(I-S)}\right)$, yields that $\lim _{n \rightarrow \infty} \frac{\left\|T^{n}\right\|}{n}=0$ by uniform ergodicity of $S$.

Since $R(I-T)=R(I-S)$ is closed, $T$ is uniformly ergodic by [8]. 
Theorem 3.3. Let $T$ be a Cesàro bounded operator on a real or complex Banach space $X$,

and $Y$ be a closed subspace of $X$ which is invariant under $T$.

If there exists $k \geq 1$ such that $R\left[(I-T)^{k}\right] \subset Y$ and $\left.T\right|_{Y}$ is uniformly ergodic, then $R(I-T)$ is closed and $X=R(I-T) \oplus N(I-T)$. Moreover, $T$ is uniformly ergodic.

\section{Proof:}

Let $T$ be a Cesàro bounded operator on a real or complex Banach space $X$, then by Proposition 2.1 we have $R(I-T) \cap N(I-T)=\{0\}$, hence $\operatorname{asc}(I-T) \leq 1$.

Let $Y \subset X$ be a closed subspace which is invariant under $T$ and denote by $S=\left.T\right|_{Y}$.

Assume that there is an integer $k \geq 1$ such that $R\left[(I-T)^{k}\right] \subset Y$ and $S$ is uniformly ergodic. Since $N(I-S) \subset N(I-T)$, then $R(I-T) \cap N(I-S)=$ $\{0\}$. Since $S$ is uniformly ergodic, then $Y=R(I-S) \oplus N(I-S)$ and $R(I-S)$ is closed. By assumption we infer that $R\left[(I-T)^{k}\right] \subset R(I-S)$.

The uniform ergodicity of $S$ implies that $I-S$ is invertible on $R(I-S)$, so $R\left[(I-S)^{k}\right]=R(I-S)$. Hence

$$
R\left[\left(I-T^{k}\right)\right] \subset R(I-S)=R\left[(I-S)^{k}\right] \subset R\left[(I-T)^{k}\right]
$$

which shows that $R\left[(I-T)^{k}\right]=R(I-S)$ and this yields $R\left[(I-T)^{n}\right]=$ $R(I-S)$ for all $n \geq k$, hence $\operatorname{des}(I-T)<\infty$. Thus by [11, Theorem V.6.2] $X=R(I-T) \oplus N(I-T)$.

Therefore, for all $n \geq 1, R(I-T)=R\left[(I-T)^{n}\right]=R(I-S)$. Consequently,

$$
M_{n}\left(\left.T\right|_{R(I-T)}\right)=M_{n}\left(\left.S\right|_{R(I-S)}\right)
$$

which implies $\frac{\left\|T^{n}\right\|}{n} \rightarrow 0$ by uniform ergodicity of $S$. Since $R(I-T)=$ $R(I-S)$ is closed, then $T$ is uniformly ergodic by [8].

Corollary 3.2. Let $T$ be a Cesàro bounded operator on a real or complex Banach space $X$. $T$ is uniformly ergodic if (and only if) the restriction of $T$ to $\overline{R\left[(I-T)^{k}\right]}$ is uniformly ergodic, for some integer $k \geq 1$. 
Now, we recall the following lemma that was introduced in $[10$, Theorem $2.2(\mathrm{ii})]$.

Lemma 3.1. Let $T$ be a Cesàro bounded operator on a complex Banach space $X$ with $\sigma(T) \cap \Gamma \subset\{1\}$, where $\Gamma$ is the unit circle, then $\lim _{n \rightarrow \infty} \frac{\left\|T^{n}\right\|}{n}=0$.

In accordance with the previous lemma, we infer from Theorem 1.1 and Theorem 3.3 the following result:

Corollary 3.3. Let $T$ be a bounded linear operator on a complex Banach space $X$.

Then the following conditions are equivalent:

1. $\left\{T^{n}\right\}_{n \geq 0}$ converge uniformly in $\mathbf{B}(X)$,

2. $T$ is uniformly ergodic and $\sigma(T) \cap \Gamma \subset\{1\}$,

3. $T$ is Cesàro bounded, $\sigma(T) \cap \Gamma \subset\{1\}$ and $R\left[(I-T)^{k}\right]$ is closed, for some integer $k \geq 1$,

4. $T$ is Cesàro bounded, $\sigma(T) \cap \Gamma \subset\{1\}$ and $\left.T\right|_{Y}$ is uniformly ergodic for some closed subspace $Y \subset X$ which is T-invariant and $R\left[(I-T)^{k}\right] \subset$ $Y$, for some integer $k \geq 1$,

5. $T$ is Cesàro bounded, $\sigma(T) \cap \Gamma \subset\{1\}$ and the restriction of $T$ to $\overline{R\left[(I-T)^{k}\right]}$, for some integer $k \geq 1$, is uniformly ergodic.

In analogy with the Gelfand-Hille theorem, we give the following theorem:

Theorem 3.4. Let $T$ be a bounded linear operator on a complex Banach space $X$ such that $\sigma(T)=\{1\}$. Let $Y \subset X$ be a closed subspace and $T$-invariant such that $R\left[(I-T)^{k}\right] \subset Y$, for some integer $k \geq 1$. If $\left.T\right|_{Y}$ is uniformly ergodic, then $(I-T)^{k+1}=0$.

Proof:

Let $S=\left.T\right|_{Y}$ and suppose that there is $k \geq 1$ such that $R\left[(I-T)^{k}\right] \subset Y$.

Assume that $S$ is uniformly ergodic, then $Y=R(I-S) \oplus N(I-S)$ and $R(I-S)$ is closed.

Since $\sigma(S) \subset \sigma(T)=\{1\}$, we have $\sigma(S)=\{1\}$. Lemma 2.3 implies $S=I$, which yields $Y=N(I-S)$. Since $R\left[(I-T)^{k}\right] \subset Y=N(I-S) \subset$ $N(I-T)$, then $R\left[(I-T)^{k+1}\right]=\{0\}$, which means $(I-T)^{k+1}=0$. 
Acknowledgments.

The authors wish to express their indebtedness to the referee, for his suggestions and valuable comments on this paper.

\section{References}

[1] P. Aiena, Fredholm and Local Spectral Theory with Applications to Multipliers, Kluwer. Acad. Press, (2004).

[2] M. Becker, A condition equivalent to uniform ergodicity, Studia Math., 167, pp. 215-218, (2005).

[3] S. R. Caradus, W. E. Pfaffenberger, B. Yood, Calkin Algebras and Algebras of Operators on Banach Spaces, Dekker, New York, (1974).

[4] N. Dunford, Spectral theory I. Convergence to projections, Trans. Amer. Math. Soc.54, pp. 185-217, (1943).

[5] S. Grabiner and J. Zemànek, Ascent, descent, and ergodic properties of linear operators, J. Operator Theory, 48 (2002), 69-81.

[6] J. J. Koliha, Convergent and stable operators and their generalizations, J. Math. Anal. Appl., 43, pp.778-794, (1993).

[7] U. Krengel, Ergodic Theorems, Walter de Gruyter Studies in Mathematics 6, Walter de Gruyter, Berlin-New York, (1985).

[8] M. Lin, On the uniform ergodic theorem, Proc. Amer. Math. Soc., 43, pp. 337-340, (1974).

[9] M. Mbekhta and J. Zemànek, Sur le théorème ergodique uniforme et le spectre, C. R. Acad. Sci. Paris sèrie I Math., 317, pp. 1155-1158, (1993).

[10] L. Suciu and J. Zemànek, Growth conditions on Cesàro means of higher order, Acta Sci. Math. (Szeged), 79, pp. 545-581, (2013).

[11] A. E. Taylor and D. C. Lay, Introduction to Functional Analysis, Wiley, New York, (1980). 
[12] K. Yosida, Mean ergodic theorem in Banach space, Proc. Imp. Acad. Tokyo 14, pp. 292-294, (1938).

[13] J. Zemànek, On the Gelfand-Hille theorems, in Functional Analysis and Operator Theory, Banach Center Publ., vol. 30, Polish Acad. Sci., Warszawa, pp. 369-385, (1994).

\section{Abdelaziz Tajmouati}

Sidi Mohamed Ben Abdellah University

Faculty of Sciences Dhar El Mahraz.

Fez

Morocco

e-mail: abdelaziztajmouati@yahoo.fr

\section{Abdeslam El Bakkali}

Chouaib Doukkali University

Faculty of Sciences

El Jadida,

Morocco

e-mail: aba0101q@yahoo.fr

and

\section{Barki Fatih}

Sidi Mohamed Ben Abdellah University

Faculty of Sciences Dhar El Mahraz.

$\mathrm{Fez}$

Morocco

e-mail: fatihbarki@gmail.com 